\title{
Numerical Evaluation of Fluid Mixing Phenomena in Boiling Water Reactor Using Advanced Interface Tracking Method*
}

\author{
Hiroyuki YOSHIDA** and Kazuyuki TAKASE $^{* *}$ \\ **Japan Atomic Energy Agency, \\ 2-4, Shirakata, Tokai, Naka, JAPAN \\ E-mail: yoshida.hiroyuki@jaea.go.jp
}

\begin{abstract}
Thermal-hydraulic design of the current boiling water reactor (BWR) is performed with the subchannel analysis codes which incorporated the correlations based on empirical results including actual-size tests. Then, for the Innovative Water Reactor for Flexible Fuel Cycle (FLWR) core, an actual size test of an embodiment of its design is required to confirm or modify such correlations. In this situation, development of a method that enables the thermal-hydraulic design of nuclear reactors without these actual size tests is desired, because these tests take a long time and entail great cost. For this reason, we developed an advanced thermal-hydraulic design method for FLWRs using innovative two-phase flow simulation technology.

In this study, a detailed Two-Phase Flow simulation code using advanced Interface Tracking method: TPFIT is developed to calculate the detailed information of the two-phase flow. In this paper, firstly, we tried to verify the TPFIT code by comparing it with the existing 2-channel air-water mixing experimental results. Secondary, the TPFIT code was applied to simulation of steam-water two-phase flow in a model of two subchannels of a current BWRs and FLWRs rod bundle. The fluid mixing was observed at a gap between the subchannels. The existing two-phase flow correlation for fluid mixing is evaluated using detailed numerical simulation data. This data indicates that pressure difference between fluid channels is responsible for the fluid mixing, and thus the effects of the time average pressure difference and fluctuations must be incorporated in the two-phase flow correlation for fluid mixing. When inlet quality ratio of subchannels is relatively large, it is understood that evaluation precision of the existing two-phase flow correlations for fluid mixing are relatively low.
\end{abstract}

Key words: Fluid Mixing Phenomena, Advanced Interface Tracking Method, Gas-Liquid Two-Phase Flow, Computational Fluid Dynamics, Boiling Water Reactor, Nuclear Engineering

\section{Introduction}

Design studies of a new generation light water reactor named innovative water reactor for flexible fuel cycle (FLWR) are underway at Japan Atomic Energy Agency (JAEA) ${ }^{(1)}$. While its plant design was based mainly on that of the current boiling water reactor (BWR), the reactor core and fuel bundle designs were altered drastically. In order to achieve a conversion ratio higher than unity without adversely affecting the safety performance, a MOX fuel and a depleted uranium fuel are used in the core and a hexagonal tight-lattice 
fuel rod bundle with about $1 \mathrm{~mm}$ of gap width were selected.

The two-phase fluid mixing behavior in fuel bundles plays an important role in the thermal-hydraulic performance of the fuel rod bundle, because it has strong effects on spatial distributions of the void fraction, quality and mass flow rate within it. The subchannel analysis method has been used for the prediction of the macroscopic thermal-hydraulic characteristics, such as critical power and pressure loss, of a wide variety of fuel rod bundle designs. In thermal-hydraulic design of the current BWR, this method evaluates the fluid mixing effects using correlations with empirical results of actual-size tests. Then, to apply this method to thermal-hydraulic design of the FLWR, an actual size test that simulates FLWRs design is required to confirm or modify the correlations.

In this situation, development of a method that enables the thermal-hydraulic design of FLWRs without these actual size tests is desired, because these tests take a long time and entail great cost. Then, we developed an advanced thermal-hydraulic design method for FLWRs using innovative two-phase flow simulation technology. One of the simulation codes: TPFIT focuses on the two-fluid mixing phenomena. Due to narrower rod gaps and the channels being surrounded by rods, bubble/slug-to-bubble/slug and bubble/slug-to-wall interactions may occur more frequently within the FLWRs core than in those of current BWRs, and these may affect the two-fluid mixing characteristics by way of the deformation, separation and coalescence of bubbles/slugs caused by the interactions. The TPFIT code could simulate these interactions directly by using an advanced interface-tracking method.

A series of verification studies on the TPFIT code has been conducted by subjecting it to experimental analyses. ${ }^{(2,3,4)}$ In this paper, firstly, we tried to verify the TPFIT code comparing with existing 2-channel air-water mixing experimental results. Secondary, we applied the TPFIT code to two-phase flow in the subchannels of current BWRs and FLWRs rod bundle.

\section{Nomenclature}

\begin{tabular}{|c|c|c|}
\hline$e$ & : & Internal energy $(\mathrm{J} / \mathrm{kg})$ \\
\hline$f$ & : & Volume fraction of fluid (-) \\
\hline$g$ & : & Gravitational acceleration $\left(\mathrm{m} / \mathrm{s}^{2}\right)$ \\
\hline$p$ & : & Static pressure $(\mathrm{Pa})$ \\
\hline$t$ & : & Time (s) \\
\hline$T$ & : & Temperature $(\mathrm{K})$ \\
\hline$u$ & : & Velocity $(\mathrm{m} / \mathrm{s})$ \\
\hline$X_{n}$ & : & Inlet quality for Ch.n(-) \\
\hline$x$ & : & Time average quality (-) \\
\hline$x, y, z$ & : & Distance $(\mathrm{m})$ \\
\hline$W$ & : & Inlet mass flow rate $(\mathrm{kg} / \mathrm{s})$ \\
\hline$w$ & : & Moved mass flow rate from Ch. 1 to $\mathrm{Ch} .2(\mathrm{~kg} / \mathrm{s})$ \\
\hline \multicolumn{3}{|c|}{ (Greek letters) } \\
\hline$\Delta p$ & : & Pressure difference between subchannels $(\mathrm{Pa})$ \\
\hline$\Gamma$ & : & Mixing coefficient (-) \\
\hline$\lambda$ & : & Thermal conductivity $(\mathrm{W} / \mathrm{m} / \mathrm{K})$ \\
\hline$\rho$ & : & Density $\left(\mathrm{kg} / \mathrm{m}^{3}\right)$ \\
\hline$\sigma$ & : & Surface tension $(\mathrm{N} / \mathrm{m})$ \\
\hline$\tau$ & : & Shear stress $(\mathrm{Pa})$ \\
\hline \multicolumn{3}{|c|}{ (Subscripts) } \\
\hline$i, j, k$ & $\cdot$ & Direction \\
\hline$m$ & : & Phase ( $l$ :liquid, $g$ :gas) \\
\hline
\end{tabular}




\section{Numerical Procedures}

\subsection{Governing Equations and Numerical Solution}

In the TPFIT code, considering the time-dependent Navier-Stokes equation for compressible flow, the conservative equations of mass, momentum and energy are described as follows;

Mass:

$$
\frac{D \rho}{D t}=-\rho \frac{\partial u_{i}}{\partial x_{i}}
$$

Momentum:

$$
\frac{D u_{i}}{D t}=-\frac{1}{\rho} \frac{\partial p}{\partial x_{i}}+\frac{1}{\rho} \frac{\partial \tau_{i j}}{\partial x_{j}}+g_{i}+\sigma_{i},
$$

Energy:

$$
\frac{D e}{D t}=-\frac{p}{\rho} \frac{\partial u_{i}}{\partial x_{i}}+\frac{1}{\rho} \frac{\partial}{\partial x_{i}}\left(\lambda \frac{\partial T}{\partial x_{i}}\right)+q,
$$

where $u, p, e$, are velocity, static pressure and internal energy. $g$ and $\sigma$ in the momentum equation are the gravity and surface tension force. Two-phase fluid density $\rho$ is calculated using the densities and the volumetric fractions of both phases:

$$
\rho=\rho_{l} f_{l}+\rho_{g} f_{g}, \quad f_{g}=1-f_{l}
$$

Subscripts $g$ and $l$ are used to represent gas and liquid phase. The momentum equation (Eq.2) is solved by the CIP (Cubic Interpolated Pseudo-particle) method ${ }^{(5)}$. The energy equation (Eq.3) is used to obtain the Poison equation of the static pressure. Temperature is estimated with fluid property routine by the static pressure and local density of both phases. The ILUCGS method is used to solve Poison equation of the static pressure. In the simulation code, Cartesian coordinate system and staggered grid are used. Surface tension in the momentum equation: $\sigma$ is estimated by use of CSF model ${ }^{(6)}$. Local densities and viscosities of liquid and gas were, of course, evaluated using solved static pressure and temperature fields by the fluid property routine.

$f_{m}$ in Eq.4 is volumetric fraction of gas or liquid phase, and calculated by the following conservative equation:

Volumetric Fraction:

$$
\frac{D f_{m}}{D t}=-f_{m} \frac{\partial u_{i}}{\partial x_{i}}-\frac{f_{m}}{\rho_{m}} \frac{D \rho_{m}}{D t},
$$

The local densities of both phases in Eqs. 4 and 5 must be evaluated to close governing equations, and calculated by use of conservative equations of mass of both phases:

Mass of both phases:

$$
\frac{D \rho_{m} f_{m}}{D t}=-\rho_{m} f_{m} \frac{\partial u_{i}}{\partial x_{i}}
$$

Eqs. 5 and 6 are calculated by the advanced interface tracking method developed in this study ${ }^{(4)}$. 
2.2 The Advanced Interface Tracking Method

The fundamental concept of the advanced interface tracking method is quite simple. That is, a transported liquid fraction between neighboring calculation control volumes during every time step is calculated through the movement of approximated gas-liquid interfaces, estimated in the Lagrangian system. Detailed formulations and their mathematical bases were described in previous papers (4), so only a procedural outline is mentioned here.

Schematic drawings of the major three operational steps within each time step in the two-dimensional case are shown in Fig.1. In the first step, as shown in Fig.1 (a), a gas-liquid interface in each control area for calculations is reconstructed in consideration of the liquid fraction represented by itself and surroundings. Here, the reconstructed interfaces within each control area are approximated using a linear function to satisfy the liquid fraction in the area by the same way as the PLIC method ${ }^{(7,8)}$. In the next step, rectangular areas within each control area are transported as following a surrounding velocity field (Fig.1 (b)). In the last step, as shown in Fig.1 (c), the liquid fraction and mass of each phase are redistributed from their relative position to between the control areas and the interfaces as well as the quadrangular areas. At the same time, the densities of each phase in each control area are calculated too.

3. Numerical Simulation of Air-Water Two-Phase Flow in Modeled 2 Subchannels

\subsection{Analysis Conditions}

The TPFIT code was applied to experimental analyses of the existing 2-channel fluid mixing experiments ${ }^{(9)}$, and comparisons between measured and calculated results were carried out to examine the capability of the code to simulate a two-phase cross flow through a narrow gap. In the experiments, the differential pressure between the subchannel at the center height of the mixing section and the exit air and water flow rate of each subchannel were measured.

Numerical analyses of air-water flow fluid mixing ("air-water cases") were applied between the length of $-100 \mathrm{~mm}$ and $+60 \mathrm{~mm}$ from the lower edge of the mixing section in the flow direction of the test channel as shown in Fig.2 (a). The flow area is divided into 
two channels by a flat plate (partition plate). At the top of the partition plate, there is a narrow slit, through which the channels are connected. The flow channel is divided into 3 parts, developing section, mixing section and outlet section. The narrow slit is located in the mixing section, and fluid mixing is occurred at this section. The developing section is set up to get developed flow at inlet of the mixing section. The outlet section is located at top of the calculated test channel to let out air-water two-phase flow smoothly.

Regular mesh division in the Cartesian system was adopted and two subchannels and the interconnection were formed by using obstacles as shown in Fig.2 (b). The calculation mesh size was set to $2 / 3 \mathrm{~mm}$ to satisfy the condition that the number of the calculation meshes of gap region must be more than 6 based on results of numerical simulation of air-water and steam-water two-channel fluid mixing tests ${ }^{(10)}$ Then, the total number of the calculation meshes was 496,800. A non-slip wall, constant exit pressure and constant inlet velocity were selected as boundary conditions for each subchannel. While surface tension was considered, the effects of the contact angle of the water on the channel walls were set to 15 degree. The time step was controlled with a typical safety factor of 0.2 to keep it lower than the limitation value given by the Courant condition and stability condition of the CSF model ${ }^{(6)}$.

Air and water were injected through the air and water inlet section located at lower part of the modeled test channel (see Fig.2 (a)). The area of the air and water inlet section was constant, and the inlet air and water velocities were varied to simulate experimental conditions. Eight cases of air-water two-phase flow simulations were performed and calculation conditions were summarized in Table 1. In the simulation, inlet liquid mass flux for two subchannels was same. Outlet pressure and inlet temperature of air and water is set to atmospheric pressure and room temperature respectively. In the case 1-1 to 4, inlet liquid mass flux was set to $370(\mathrm{~kg} / \mathrm{s})$, and inlet quality was relatively low. In the case 1-5 to 8 , inlet liquid mass flux was set to $277(\mathrm{~kg} / \mathrm{s})$, and inlet quality was relatively high. Then, we describe case 1-1 to 4 with "low quality cases", and case 1-5 to 8 with "high quality cases".

\subsection{Results and discussion}

Example of the calculated slug behavior in the test channel is shown in Fig.3. In the 
Fig.3, the calculated gas-liquid interfaces are defined as isosurface at a void fraction of $50 \%$. As shown in Fig.3, the fluid mixing was observed at a gap between the subchannels.

The measured and calculated differential pressure between two subchannels is shown in Fig.4. The pressure differences were estimated at the center height of the mixing section as same as the experiment. In the figure, time average values ("Average") and standard deviation of fluctuating values ("Fluctuation" in the figure) of differential pressure are shown. The fluctuating values were evaluated in the following equation:

$$
\sigma_{\Delta p}=\sqrt{\left(\Delta p_{T}-\Delta p_{S}\right)^{2}}
$$

where,

$$
\Delta p_{S}=\overline{\Delta p_{T}}
$$

$\Delta P_{T}$ : instantaneous value of differential pressure.

$\Delta P_{S}$ : time average value of differential pressure

Tendencies of overestimate were seen in the results of fluctuating values of low quality case (Fig.4 (a)). In the experimental results, the fluctuating value was gradually increased according to increase of the inlet quality ratio. However, in the numerical results, the fluctuating values were almost constant. These overestimations of the fluctuating values and difference of inlet quality ratio effects on the fluctuating values were caused by the slug (bubble) breakup at the mixing section and water properties routine used in TPFIT code. This properties routine underestimates sonic speed in water, and the pressure wave generated by slug breakup was enhanced in the subchannels. Therefore, pressure wave in

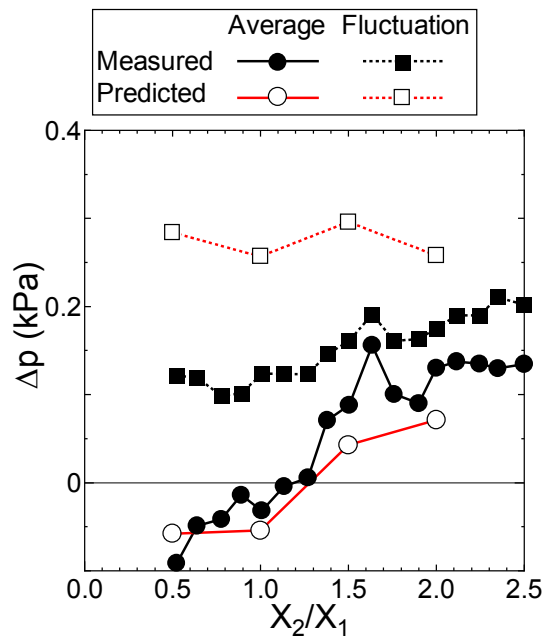

(a) Low quality case (Case 1-1 4)

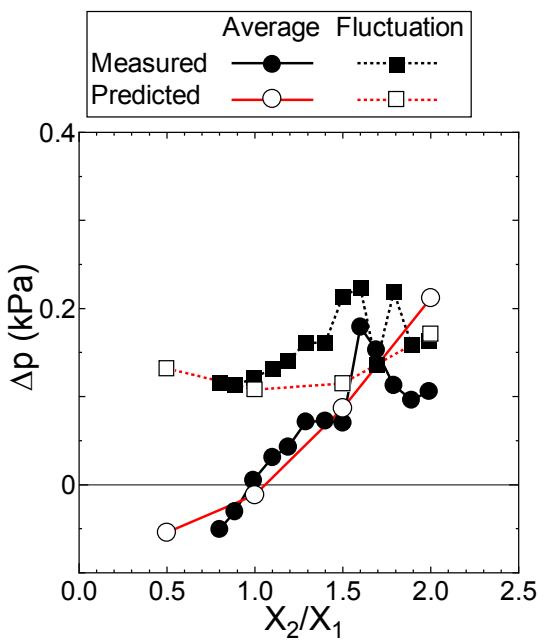

(b) High quality case (Case 1-5 8)

Fig.4 Measured and calculated pressure difference between subchannels 


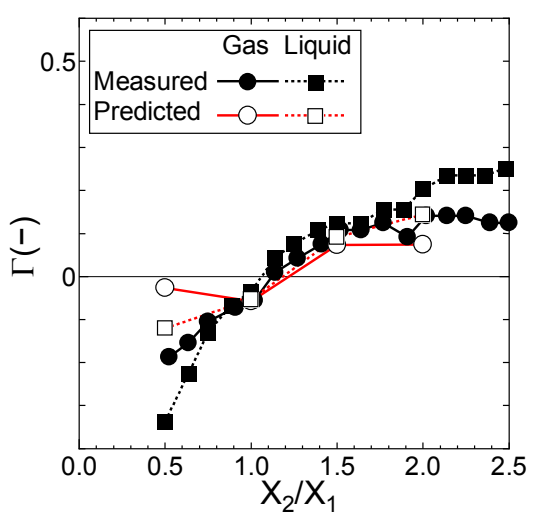

(a) Low quality case (Case 1-1 4)

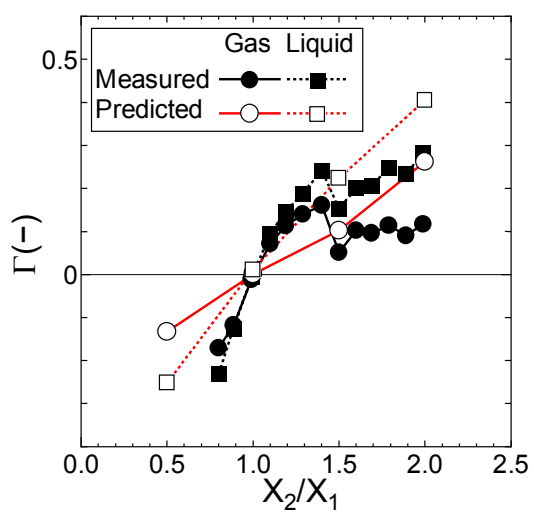

(b) High quality case (Case 1-5 8)

Fig.5 Measured and calculated mixing coefficient

the subchannels was overestimated in the subchannels. In the low quality case, slug breakup occurred more frequently, then, overestimations of the fluctuating values were observed. The predicted time average values of low quality case almost agree with the experimental results both qualitatively and quantitatively.

In the cases of the inlet quality ratio relatively low $\left(X_{2} / X_{1}<1.5\right)$, the predicted time average and fluctuating values of high quality cases almost agree with the experimental results (Fig.4 (b)). However, in the cases of the inlet quality ratio relatively high $\left(1.5<X_{2} / X_{1}\right)$, the predicted values did not agree with the measured values. In the experimental results, the time average and fluctuating values took the maximum value at around $X_{2} / X_{1}=1.6$, and gradually decreases according to increase of inlet quality ratio. In addition, in the experimental results at $X_{2} / X_{1}=1.7$, time average value was greater than fluctuating value. In the numerical results, time average value was greater than fluctuating value at $X_{2} / X_{1}=2.0$. In the result, in the cases of inlet quality ratio relatively high, effects of inlet quality ratio on average and fluctuating pressure difference were underestimated. In the interface tracking method (including the advanced interface tracking method), only single interface can exist in one numerical cell. Then, when several interfaces approached distance less than numerical cell size, those interfaces are evaluated as single coalescent interface. Therefore, occurrences of bubbles coalescence depend on spacial resolution of calculation channel. It is thought that the influence of the entrance quality ratio $\left(X_{2} / X_{1}\right)$ was underestimated by influence of overestimation of bubble coalescence caused by insufficient spatial resolution.

The measured and calculated mixing coefficients of both phases are shown in Fig.5. The mixing coefficients of gas (air or steam) and liquid (water) are defined as follows ${ }^{(9)}$ :

$$
\Gamma_{m}=\frac{w_{m}}{W_{m 1}+W_{m 2}}
$$

where,

$W_{m 1}$ : Inlet mass flow rate of $m$ phase for Ch.1

$W_{m 2}$ : Inlet mass flow rate of $m$ phase for Ch.2

$w_{m}$ : Moved mass flow rate from Ch.1 to $\mathrm{Ch} .2$

The negative value of mixing coefficients means gas or liquid moved from Ch.2 to Ch.1.

In the cases of inlet quality ratio relatively low $\left(X_{2} / X_{1}=0.5\right)$, tendencies of underestimate of the mixing coefficients were seen. These underestimations of the mixing coefficients corresponded to the underestimation of the time average pressure difference and the overestimation of the fluctuating pressure difference. The fluctuating pressure difference restrains fluid mixing ${ }^{(9)}$, and then the overestimation of the fluctuating pressure differences leads underestimation of the mixture coefficient. In the cases of inlet quality ratio relatively high $\left(X_{2} / X_{1}>1.5\right)$, the predicted mixing coefficients of high quality cases overestimated the experimental results. These differences between measured and predicted values of the 
Table 2 Calculation conditions for fluid mixing phenomena in BWRs fuel bundle

\begin{tabular}{|c|c|c|c|c|}
\hline \multirow[t]{2}{*}{ Case } & \multicolumn{2}{|c|}{$\begin{array}{l}\text { Inlet liquid mass } \\
\text { flux }\left(\mathrm{kg} / \mathrm{m}^{2} \mathrm{~s}\right)\end{array}$} & \multicolumn{2}{|c|}{$\begin{array}{l}\text { Inlet quality } \\
\qquad(\%)\end{array}$} \\
\hline & Ch.1 & Ch.2 & Ch.1 & Ch.2 \\
\hline $2-1$ & \multirow{8}{*}{\multicolumn{2}{|c|}{1400}} & 0.0067 & \multirow{4}{*}{0.0264} \\
\hline $2-2$ & & & 0.0134 & \\
\hline $2-3$ & & & 0.0264 & \\
\hline $2-4$ & & & 0.0513 & \\
\hline $2-5$ & & & 0.0184 & \multirow{4}{*}{0.0362} \\
\hline 2-6 & & & 0.0362 & \\
\hline $2-7$ & & & 0.0533 & \\
\hline $2-8$ & & & 0.0699 & \\
\hline
\end{tabular}

Table 3 Calculation conditions for fluid mixing phenomena in FLWRs fuel bundle

\begin{tabular}{|c|c|c|c|c|c|}
\hline \multirow[t]{2}{*}{ Case } & \multirow[t]{2}{*}{$\begin{array}{l}\text { Gap width } \\
\text { (mm) }\end{array}$} & \multicolumn{2}{|c|}{$\begin{array}{l}\text { Inlet liquid } \\
\text { mass flux } \\
\left(\mathrm{kg} / \mathrm{m}^{2} \mathrm{~s}\right)\end{array}$} & \multicolumn{2}{|c|}{$\begin{array}{c}\text { Inlet } \\
\text { quality } \\
(\%)\end{array}$} \\
\hline & & Ch.1 & Ch. 2 & Ch.1 & Ch.2 \\
\hline $3-1$ & \multirow{4}{*}{1.3} & \multirow{4}{*}{600} & 150 & \multirow{4}{*}{0.05} & 0.21 \\
\hline $3-2$ & & & & & 0.002 \\
\hline $3-3$ & & & 600 & & 0.03 \\
\hline $3-4$ & & & & & 0.08 \\
\hline $3-5$ & \multirow{4}{*}{1.0} & \multirow{4}{*}{600} & \multirow{4}{*}{600} & \multirow{4}{*}{0.05} & 0.05 \\
\hline $3-6$ & & & & & 0.03 \\
\hline $3-7$ & & & & & 0.08 \\
\hline $3-8$ & & & & & 0.12 \\
\hline
\end{tabular}

mixing coefficients corresponded to those of the pressure difference.

\section{Evaluation of Existing Correlations for Fluid Mixing Phenomena}

\subsection{Analysis Conditions}

To evaluate the existing two-phase flow correlation for fluid mixing phenomena, two-phase flow in 2 modeled subchannels for BWRs and FLWRs fuel bundles were performed ("BWR cases" and "FLWR cases"). For this, 16 cases of two-phase flow simulations were performed (see table 2 and 3). In the tight-lattice rod bundles, mass flux ratio between center subchannels and peripheral subchannels is thought to be larger than that in the current BWR rod bundles. To evaluate the effects of mass flux ratio in tight-lattice rod bundle, inlet mass flux for Ch.2 of Case 3-1 was set to one-quarter of other cases.

The calculated test channel is shown in Fig.6 (a) and Fig.7 (a). The flow area is divided into two channels by a flat plate (partition plate) as same as the air-water cases. The flow channel is divided into 2 parts, developing section and mixing section. Liquid phase velocity in the BWR cases and the FLWR cases is relatively higher than that in air-water cases. To remove the effects of two-phase flow development on fluid mixing, length of the developing section and the mixing section were extended. In addition, because effects of the outlet section were relatively small for large water velocity cases, we decided to remove the outlet section to reduce computational time.

Regular mesh division in

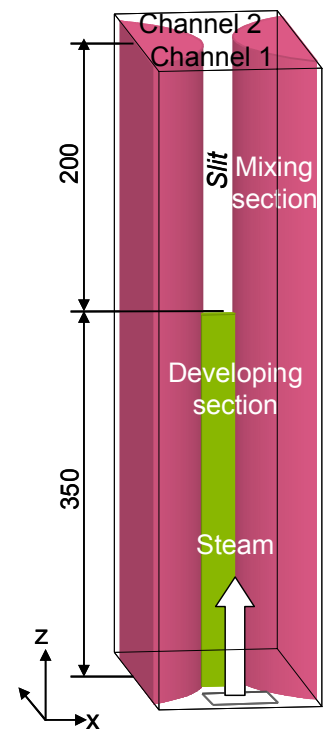

(a) Calculated test channel

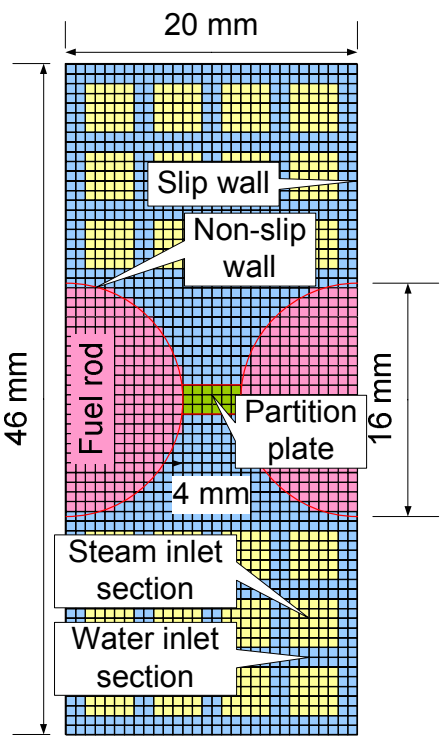

(b) Calculation mesh

Fig.6 Dimensions of calculated test channel and calculation meshes in channel cross section for BWR cases. 
the Cartesian system was adopted except for lower part of the developing section. In the lower part of developing section ( $\mathrm{z}=0 \sim 200 \mathrm{~mm}$ for BWR cases, $\mathrm{z}=0 \sim 150 \mathrm{~mm}$ for FLWR cases), to save computational resources, a relatively coarse computational meshes were used $(\Delta \mathrm{z}=2 \mathrm{~mm}$ for BWR cases and $\Delta \mathrm{z}=0.375 \mathrm{~mm})$. The other region, $\Delta \mathrm{z}$ equals to $1 \mathrm{~mm}$ for $\mathrm{BWR}$ cases and $0.25 \mathrm{~mm}$ for FLWR cases. Two subchannels and the interconnection were formed by using obstacles as same as the air-water cases. The calculation mesh sizes in horizontal planes were set to $2 / 3 \mathrm{~mm}$ for BWR cases, $1.3 / 6 \mathrm{~mm}$ for FLWR
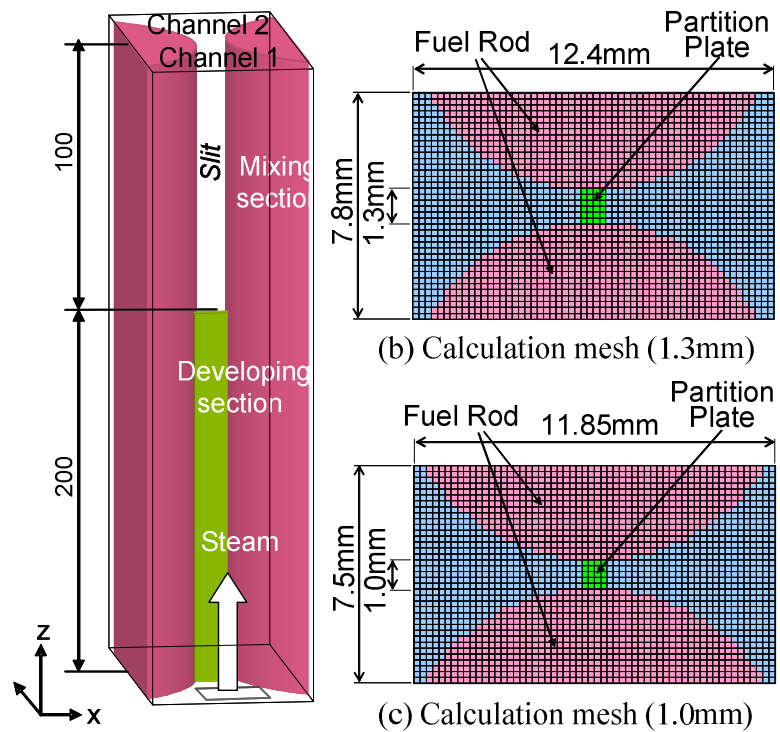

(b) Calculation mesh (1.3mm)

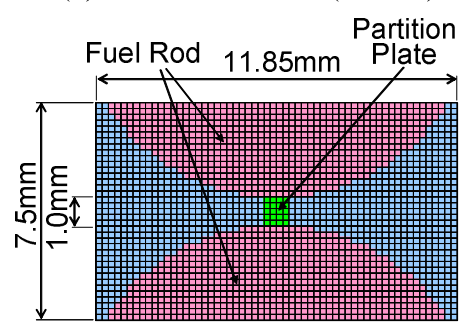

(c) Calculation mesh (1.0mm)

(a) Calculated test channel

Fig.7 Dimensions of calculated test channel and calculation meshes in channel cross section for FLWR cases.

cases $($ gap $=1.3 \mathrm{~mm})$ and $1 / 6 \mathrm{~mm}$

for FLWR cases (gap=1.0 mm) to satisfy the condition that the number of the calculation meshes of gap region must be more than 6 as same as air-water cases (see Fig.2(b)).

A non-slip wall, constant exit pressure and constant inlet velocity were selected as boundary conditions for each subchannel. To simulate the operating conditions of the BWR and the FLWR, outlet pressure and inlet temperature of steam and water is set to $7 \mathrm{MPa}$ and saturation temperature at $7 \mathrm{MPa}$ respectively. Steam and water were injected through the steam and water inlet section located at lower part of the modeled test channel. The steam and water inlet sections were optimized to get a developed flow at inlet of test section, and divided into twelve small sections for BWR cases.

\subsection{Results and Discussion}

Example of the calculated behavior in the test channel and detail of slug behavior of steam-water cases are shown in Fig.8. As shown in Fig.8, the fluid mixing between Ch.1 and Ch.2 was observed at a gap between the subchannels. Though inlet quality of both subchannels were equivalent in this case (inlet quality ratio $\left(X_{2} / X_{1}\right)$ was equal to 1.0$)$, fluid

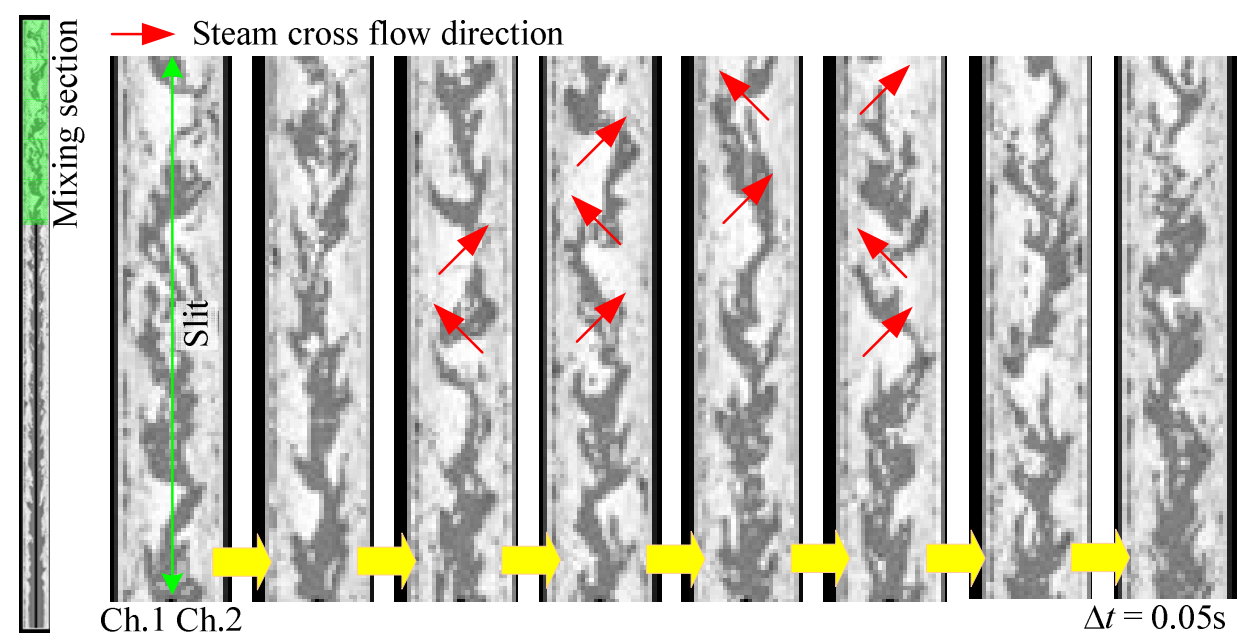

Fig.8 Calculated slug behavior of case 3-5 in mixing section 
mixing occurred between two subchannels.

The existing two-phase flow correlation for fluid mixing (fluctuating pressure model ${ }^{(9)}$ ) was evaluated using detailed numerical simulation data. The fluctuating pressure model is expressed as follows:

$$
\left|w_{T}\right|=\sqrt{\frac{2 \rho_{l}\left|\Delta p_{T}\right|}{K(1-x)^{2}\left[1+B x\left(\frac{\rho_{l}}{\rho_{g}}-1\right)\right]}}=f(x) \sqrt{\left|\Delta p_{T}\right|}
$$

where,

$w_{T}$ : evaluated moved mass flow rate by the fluctuating pressure model $[\mathrm{kg} / \mathrm{s}]$

$K$ : time average pressure loss coefficient [-]

$x:$ time average quality [-]

$B$ : time average two-phase flow pressure loss coefficient $[-]$

By the fluctuating pressure difference model, mixing coefficients for both phases are evaluated by following equations.

$$
\Gamma_{m, T}=\frac{\overline{w_{m, T}}}{W_{m 1}+W_{m 2}}
$$

where,

$$
\begin{aligned}
& w_{l, T}=-(1-x) f(x) \frac{\Delta p_{T}}{\sqrt{\left|\Delta p_{T}\right|}} \\
& w_{g, T}=-x f(x) \frac{\Delta p_{T}}{\sqrt{\left|\Delta p_{T}\right|}}
\end{aligned}
$$

In above equations, instantaneous pressure difference values and time average values (pressure loss coefficient, quality and two-phase flow pressure loss coefficient) are evaluated by numerical results.

To estimate effects of fluctuating pressure on the mixing coefficients, the mixing coefficients using time average pressure difference were also evaluated for BWR cases:

$$
\Gamma_{m, S}=\frac{w_{m, S}}{W_{m 1}+W_{m 2}}
$$

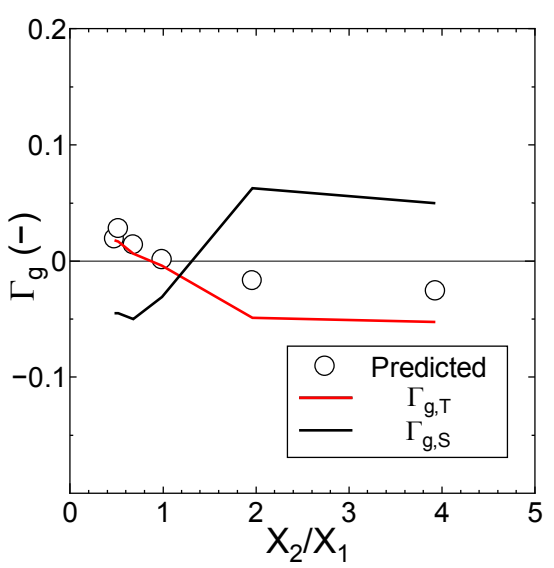

(a) Gas

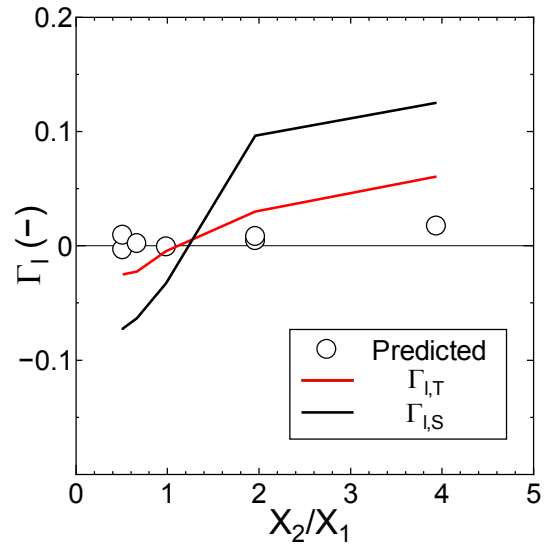

(b) Liquid

Fig.9 Evaluation of the fluctuating pressure model for BWR cases (Case 2-1 8). 
where,

$$
\begin{aligned}
& w_{l, S}=-(1-x) f(x) \frac{\Delta p_{S}}{\sqrt{\left|\Delta p_{S}\right|}} \\
& w_{g, S}=-x f(x) \frac{\Delta p_{S}}{\sqrt{\left|\Delta p_{S}\right|}}
\end{aligned}
$$

Evaluated mixing coefficients by fluctuating pressure model, $\Gamma_{m, T}$ are shown in Fig.9. Predicted mixing coefficients by TPFIT code and $\Gamma_{m, S}$ are also shown in figures. The evaluated mixing coefficients, $\Gamma_{m, T}$ were in reasonable agreement with the predicted results. Evaluated mixing coefficients by use of time average pressure difference, $\Gamma_{m, S}$ overestimated the predicted results in almost all cases, and it is understood that the fluctuating component of pressure difference restrains the fluid mixing between subchannels.

Evaluated total mixing coefficients $\left(\Gamma_{l}+\Gamma_{g}\right) \quad$ by fluctuating pressure model and conventional fluid mixing model ${ }^{(11)}$ for FLWR cases are shown in Fig.10. Predicted mixing coefficients by the TPFIT code are also shown in figures. The evaluated mixing coefficients by fluctuating pressure model for relatively low inlet quality ratio cases $\left(X_{2} / X_{1}<2\right)$ were in reasonable agreement with the predicted results for both $1.3 \mathrm{~mm}$ and 1.0 $\mathrm{mm}$ gap spacing. However, evaluated mixing coefficients by conventional fluid mixing model were different from predicted mixing coefficients by the TPFIT code both qualitatively and quantitatively. Evaluated mixing coefficients for relatively high inlet quality ratio cases $\left(X_{2} / X_{1}>2\right)$ by fluctuating pressure model and conventional fluid mixing model showed underestimation and overestimation respectively.

\section{Conclusion}

The TPFIT, which was a detailed gas-liquid two-phase flow simulation code based on the advanced interface tracking method, was applied to experimental analyses of the existing 2-channel air-water mixing tests, and comparisons between measured and calculated results were carried out to examine the capability of the code to simulate two-phase cross flow through a narrow gap. The calculated difference pressure between subchannels and the mixing coefficients for both phases agree with the measured values. However, effects of the spatial resolution on fluid mixing phenomena must be examined 
more.

The TPFIT code applied to numerical simulation of fluid mixing between two subchannels on current BWR and FLWR operating conditions. The existing two-phase flow correlations for the fluid mixing were evaluated using detailed numerical simulation data. From the data, pressure difference between fluid channels is responsible for the fluid mixing, and effects of the fluctuating pressure difference must be incorporated in the two-phase flow correlation for fluid mixing. When inlet quality ratio of subchannels is relatively large, evaluated mixing coefficients by existing two-phase flow correlations for fluid mixing are different from those of the detailed numerical simulation data.

\section{Acknowledgments}

This paper contains some results obtained within the task "Development of Fuel Assembly for Very High Burn-up Water-cooled Breeding Reactor" entrusted from the Ministry of Education, Culture, Sports, Science and Technology of Japan. This research was conducted using a supercomputer of the Japan Atomic Energy Agency.

\section{References}

(1) Uchikawa, S. et al., 2005, "Investigation on Innovative Water Reactor for Flexible Fuel Cycle (FLWR) (1) Conceptual Design”, Proc. GLOBAL2005, 358, Tsukuba, Japan, Oct. 12.

(2) Yoshida, H. et al., 2004, "Verification of a Detailed Two-Phase Flow Analysis Code Based on an Advanced Interface Tracking Method(I) - Detail of Numerical Method and Analysis of Liquid Film Falling Down on Inclined Flat Plate -", Proc. of NUTHOS-6, Nara, Japan, October 4-8, N6P26.

(3) Yoshida, H. et al., 2005, "Numerical Simulation of Single Bubbles Rising Through Subchannels with Interface Tracking Method", Proc of NURETH11, Avignon, France, October 2-6, 436

(4) Yoshida, H. et al., 2006, "Development of Analytical Procedures on Two-Phase Flow in Tight-Lattice Fuel Bundles for Innovative Water Reactor for Flexible Fuel Cycle(FLWR)", Nucl. Eng. Technol., 38[2], pp.119-12

(5) Yabe, T. and Aoki, T., 1991, "A Universal Solver for Hyperbolic Equations by Cubic-Polynomial Interpolation I. One-Dimensional Solver," Comput. Phys. Commun., 66, pp. 219-232.

(6) Brackbill, J. U. et al., 1992, “A Continuum Method for Modeling Surface Tension”, J. Comput. Phys., 100, pp. 335-354.

(7) Youngs, D. L., 1982, "Time Dependent Multimaterial Flow with Large Fluid Distortion", in Numerical Methods for Fluid Dynamics, ed. Morton, K.M. and Baines, M.J., Academic Press, pp.27-39.

(8) Gueyffier, D. et al., 1999, "Volume-of-Fluid Interface Tracking with Smoothed Surface Stress Methods for Three-Dimensional Flows”, J. Comput. Phys., 152, pp. 423-456.

(9) Sumida, et al., "Investigation of Two-Phase Flow Mixing Between Two Subchannels", JSME Journal B, 63, 612, (1997) [in Japanese]

(10) Yoshida, H. et al., 2008, "Development of Design Technology on Thermal-Hydraulic Performance in Tight-Lattice Rod Bundles: III - Numerical Evaluation of Fluid Mixing Phenomena using Advanced Interface-Tracking Method -", J. Power and Energy Systems, 2 [1], pp.250-261

(11) Kelly, J.E. and Kazimi, M.S., "Development of the two-fluid multidimensional code THERMIT for LWR analysis", AIChE, Symposium Series, pp. 149-162 (1980). 